\title{
CONSTRUCTION OF MANIFOLDS OF POSITIVE SCALAR CURVATURE
}

\author{
RODNEY CARR
}

\begin{abstract}
We prove that a regular neighborhood of a codimension $\geq 3$ subcomplex of a manifold can be chosen so that the induced metric on its boundary has positive scalar curvature. A number of useful facts concerning manifolds of positive scalar curvature follow from this construction. For example, we see that any finitely presented group can appear as the fundamental group of a compact 4-manifold with such a metric.
\end{abstract}

0. Outline of results. We give a new method for constructing complete Riemannian manifolds of positive scalar curvature and use it to continue the investigation of properties of positive scalar curvature.

Our construction uses the idea that manifolds having spheres of dimension $\geq 2$ as "factors" will admit metrics of positive scalar curvature if the spheres can be made to carry sufficient positive curvature to dominate any negative curvature. Most of the known methods for constructing manifolds of positive scalar curvature employ this same idea. For example, any manifold of the form $M \times S^{2}$ can be given a warped-product metric of positive scalar curvature by suitably adjusting the radius of the $S^{2}$-factor. Similarly, by deforming the standard metric on $S^{3}-$ point $\}$ in a small neighborhood of the point and using the $S^{2}$-factor to carry positive curvature around the corner we can construct a complete metric of positive scalar curvature on $\mathbf{R}^{3}$. This same idea was used by Gromov and Lawson $[\mathbf{G L}]$ and Schoen and Yau $[\mathbf{S Y}]$ in proving that codimension $\geq 3$ surgeries on a manifold of positive scalar curvature yields a manifold which also carries positive scalar curvature. In this paper we generalize the above techniques to cover any manifold formed as the boundary of a regular neighborhood of a subcomplex $K$ of a manifold $M$. If the codimension of $K \geq 3$ this boundary looks locally like $K \times S^{2}$, and so should carry positive scalar curvature.

THEOREM 1. Let $M$ be an n-dimensional Riemannian manifold with a fixed smooth cell decomposition and $K$ a codimension $q \geq 3$ subcomplex of $M$. Then there is a regular neighborhood $U$ of $K$ in $M$ so that the induced metric on the boundary $\partial U$ has positive scalar curvature.

An easy consequence of this theorem is the following.

COROLLARY 2. Let $\pi$ be a finitely presented group. Then there exists a compact 4-manifold $M$ of positive scalar curvature with $\pi_{1}(M)=\pi$.

This fact is interesting since it is generally believed that manifolds that are "large" in some sense should not admit metries of positive curvature. Corollary 2

Received by the editors September 25, 1985 and, in revised form, July 17, 1986.

1980 Mathematics Subject ('lassification (1985 Revision). Primary 53C20. 
shows that in dimensions $\geq 4$ any notion of "large" which is sufficiently strong to prohibit positive scalar curvature cannot be given solely in terms of the fundamental group.

We also use Theorem 1 to investigate the structure of $R^{+}(M)$, the space of positive scalar curvature metrics on a manifold $M$. To do this we need the following.

THEOREM 3. Let $K$ be a codimension $q \geq 3$ subcomplex of a Riemannian manifold $M$. Let $U \subset M$ be a regular neighborhood of $K$. Then $U$ carries a metric of positive scalar curvature which is a product near the boundary.

Suppose $X_{1}$ and $X_{2}$ are manifolds of positive scalar curvature with boundaries diffeomorphic to $M, \partial X_{1} \cong \partial X_{2} \cong M$, so that the metrics are products near the boundaries. If the corresponding metrics on $M$ are in the same connected component of $R^{+}(M)$ we prove that $X_{1} \cup_{\phi} X_{2}$ also admits a metric of positive scalar curvature. However, we find in some cases that $\hat{A}\left(X_{1} \cup_{\phi} X_{2}\right) \neq 0$, and hence conclude that the metrics on $M$ must fall in different connected components of $R^{+}(M)$. For $M=S^{4 m-1}$ we do this using the method of plumbing disc bundles over spheres and prove the following.

THEOREM 4. The space of positive scalar curvature metrics on $S^{4 m-1}, m \geq 2$, has an infinite number of connected components.

This work is based on part of the author's doctoral dissertation. I would like to express the gratitude I owe to Professor H. Blaine Lawson for suggesting this problem and for his continual guidance.

1. Proof of Theorem 1. The neighborhood $U$ is constructed by successively forming tubular neighborhoods $U_{p}$ about the $p$-skeletons $K^{p}$ of $K, p=1, \ldots, n-$ $q$, so that at each step the induced metric on the boundary has positive scalar curvature. We construct $U_{p}$ from $U_{p-1}$ by "pulling out" around the $p$-cells of $K$, and finally obtain $U=U_{n-q}$.

To begin, we form $U_{0}$, a regular neighborhood of $K^{0}=\left\{v_{1}, \ldots, v_{r}\right\}$, as follows. For $\varepsilon>0$ let $B_{p}(\varepsilon)=\left\{x \in M \mid \operatorname{dist}_{M}(p, x) \leq \varepsilon\right\}$ be a small ball centered at $p$ and $S_{p}(\varepsilon)$ its boundary. Note that for $\varepsilon$ small the principal curvatures of $S_{p}(\varepsilon)$ are close to those of the usual sphere, that is, of the form $-1 / \varepsilon+O(\varepsilon)$. So set $U_{0}=B_{v_{1}}\left(\varepsilon_{1}\right) \cup \cdots \cup B_{v_{n}}\left(\varepsilon_{n}\right)$ where the $\varepsilon_{i}$ are chosen so that

(i) $S_{v_{i}}\left(\varepsilon_{i}\right) \cap S_{v_{j}}\left(\varepsilon_{j}\right)=\varnothing$ for $i \neq j$,

(ii) $S_{v_{i}}\left(\varepsilon_{i}\right)$ has positive scalar curvature for $i=1, \ldots, r$,

(iii) each 1-cell of $K$ coming into $v_{i}$ intersects $S_{v_{i}}\left(\varepsilon_{i}\right)$ transversely in one point.

For the inductive step assume that $U_{p-1}$ is a regular neighborhood of $K^{p-1}$ so that

(a) the metric induced on the hypersurface $H=\partial U_{p-1}$ has positive scalar curvature, and

(b) each $p$-cell $\sigma$ of $K$ which intersects $H$ does so transversely in a (p-1)-sphere.

Consider any $p$-cell and fix a diffeomorphism $\psi: \sigma \cap H \rightarrow S^{p-1}$. Let $\eta$ be the outward unit normal to $H$. By altering $K$ a little, if necessary, we can assume that

$$
\exp _{\psi_{\sigma}^{-1}(y)}(t \eta) \in \sigma, \quad t \in(-\delta, \delta),
$$

for $\delta>0$ sufficiently small and $y \in S^{p-1}$. 


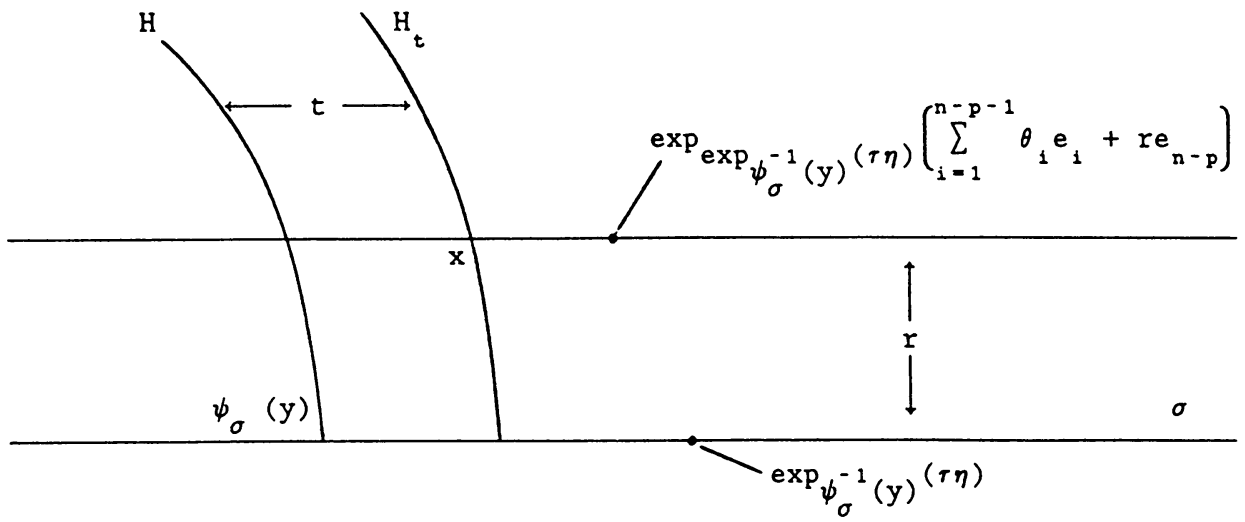

FIGURE 1

Define distance functions $\bar{t}: M \rightarrow \mathbf{R}$ and $\bar{r}: M \rightarrow \mathbf{R}^{+}$by

$$
\bar{t}(x)=\operatorname{dist}_{M}(x, H),
$$

where we measure distance positively if $x \in M$ is in the $\eta$ direction and negatively if $x$ is in the $-\eta$ direction, and

$$
\bar{r}(x)=\operatorname{dist}_{M}(x, \sigma) .
$$

Let $\left\{e_{i}\right\}_{i=1}^{n-p}$ be orthonormal sections of the mormal bundle of $\sigma$, with $e_{n-p}=$ $\operatorname{grad}(\bar{r})$. For $r>0$ set $D^{n-p}(r)=\left\{x \in \mathbf{R}^{n-p} \mid\|x\| \leq r\right\}$. For sufficiently small $R>0$ and $0<T<\delta$ identify the set

$$
V_{R, T} \equiv\{x \in M \mid \bar{r}(x) \leq R, 0 \leq \bar{t}(x) \leq r\}
$$

with $D^{n-p}(R) \times[0, T] \times S^{p-1}$ by using the map which sends $x \in V_{R, T}$ to

$$
\left(\theta_{1}, \ldots, \theta_{n-p-1}, r, t, y\right)
$$

where $\left(\theta_{1}, \ldots, \theta_{n-p-1}, r\right) \in D^{n-p}(R), t \in[0, T]$ and $y \in S^{p-1}$ are such that $H_{t} \cap$ $\sigma_{(\theta, r), y}=\{x\}$. Here $H_{t}$ is the hypersurface a constant distance $t$ from $H$ and

$$
\sigma_{(\theta, r), y}=\left\{\exp _{\exp _{\psi_{\sigma}^{-1}(y)}(\tau \eta)}\left(\sum_{i=1}^{n-p-1} \theta_{i} e_{i}+r e_{n-p}\right) \mid \tau \in(-\delta, \delta)\right\}
$$

is a curve a constant distance $r$ from $\sigma$. (See Figure 1.)

We now follow a procedure similar to that given in [GL]. In $V_{R, T}$ we wish to choose a hypersurface $H^{\prime}$ of the form

$$
H^{\prime}=\left\{\left(\theta_{1}, \ldots, \theta_{n-p-1}, r, t\right) \mid r=\gamma(t)\right\}
$$

where $\gamma$ is a curve having a graph as shown in Figure 2 so that the induced metric on $H^{\prime}$ has positive scalar curvature.

The important points about $\gamma$ are that it starts at $t=0$ and ends up as a constant $\varepsilon_{1}>0$. $H^{\prime}$ then has the property that it is "pulled out" from $H=H_{0}$ and ends up as a tube a constant distance $\varepsilon_{1}$ from the cell $\sigma$.

For the purposes of calculating the scalar curvature of $H^{\prime}$ it is convenient to introduce the frame field $\left\{e_{i}\right\}_{i=1}^{n}$ on

$$
V_{R, T} \cong D^{n-p}(R) \times[0, T] \times S^{p-1}
$$




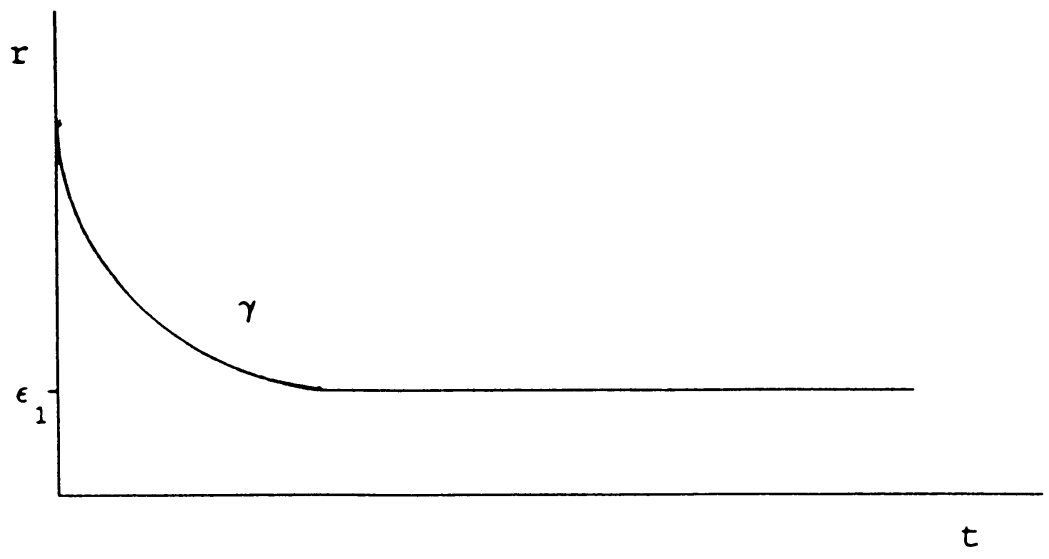

FIGURE 2

where $e_{1}, \ldots, e_{n-p}$ are, as before, given by polar coordinates about $\sigma, e_{n-p+1}=$ $\operatorname{grad}(\bar{t})$ and $\left\{e_{n-p+2}, \ldots, e_{n}\right\}$ forms an orthonormal frame field for the $S^{p-1}$-factor orthogonal to $\left\{e_{1}, \ldots, e_{n-p+1}\right\}$. Note that

$$
e_{n-p+1}=\partial / \partial t+O(r) e_{n-p} .
$$

In terms of the above basis the metric on $V_{R, T}$ has the form

$$
\left(g_{i j}\right)=\left(\begin{array}{c|c|c}
\delta_{i j} & O(r) & 0 \\
\hline O(r) & 1 & 0 \\
\hline 0 & 0 & \delta_{i j}
\end{array}\right), \quad \begin{aligned}
& i \leq n-p, \\
& \quad n-p+2 \leq i \leq n,
\end{aligned}
$$

and hence the connection has the form

$$
\begin{array}{ll}
\nabla_{e_{1}} e_{i}=-\frac{1}{r} \cdot e_{n-p}+\sum_{k=1}^{n} O(1) e_{k}, & 1 \leq i \leq n-p-1, \\
\nabla_{e_{2}} e_{n-p}=\frac{1}{r} \cdot e_{n-p}+\sum_{k=1}^{n} O(1) e_{k}, & 1 \leq i \leq n-p-1, \\
\nabla_{e_{2}} e_{j}=\sum_{k=1}^{n} O(1) e_{k}, \quad \text { otherwise. } &
\end{array}
$$

Also in terms of $\left\{e_{i}\right\}_{i=1}^{n}$, a basis $\left\{f_{i}\right\}_{i=1}^{n-1}$ for the tangent space of $H^{\prime}$ is given as

$$
f_{i}=\left\{\begin{array}{l}
\frac{e_{i}+O(1) \gamma(t) \gamma^{\prime}(t) e_{n-p}}{\left(1+O(1) \gamma(t)^{2} \gamma^{\prime}(t)^{2}\right)^{1 / 2}}, \quad i=1, \ldots, n-p-1, \\
\frac{e_{n-p-1}+\gamma^{\prime}(t) e_{n-p}}{\left(1+\gamma^{\prime}(t)^{2}\right)^{1 / 2}}, \quad i=n-p, \\
e_{i+1}, \quad i=n-p+1, \ldots, n-1,
\end{array}\right.
$$

and the unit normal

$$
\xi=\frac{e_{n-p}-\gamma^{\prime}(t) e_{n-p+1}+\sum_{k=1}^{n-p+1} O\left(\gamma(t) \gamma^{\prime}(t)\right) e_{n-p}}{\left(1+\gamma^{\prime}(t)^{2}+O\left(\gamma(t)^{2} \gamma^{\prime}(t)^{2}\right)^{1 / 2}\right.} .
$$

These expressions are complicated by terms which compensate for the fact that since the hypersurfaces $H_{t}$ are not totally geodesic the angular vectors $e_{i}, i=$ $1, \ldots, n-p-1$, fail to be tangent to $H^{\prime}$. However, we can simplify the formulas 
considerably by noting that it is possible to choose an initial segment of the curve $\gamma$ as shown in Figure 3 while retaining positive scalar curvature (since the condition of having positive scalar curvature is open) so that we can assume $\gamma^{\prime}$ is bounded and estimate the scalar curvature solely in terms of $\gamma(t)$. We need to control $\gamma^{\prime \prime}(t)$, however.

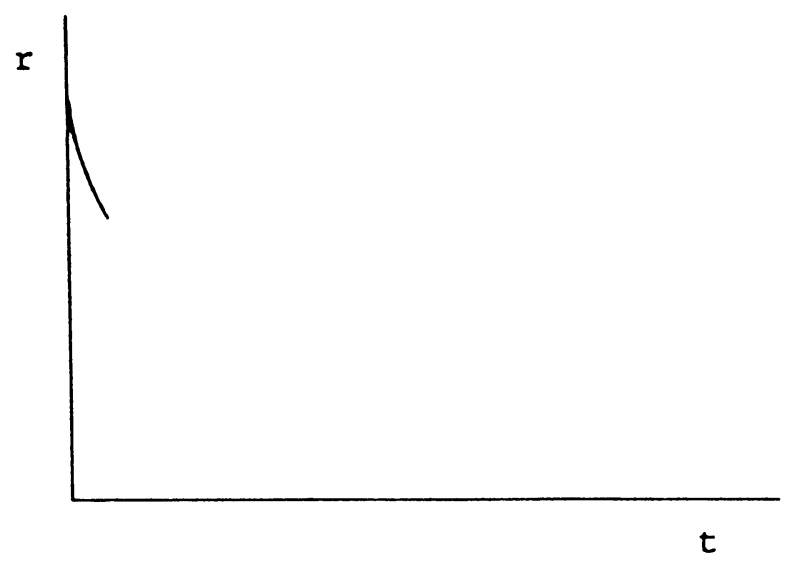

FIGURE 3

The Gauss equation

$$
K_{H^{\prime}}\left(f_{i} \wedge f_{j}\right)=K_{M}\left(f_{i} \wedge f_{j}\right)+g\left(\nabla_{f_{i}} \xi, f_{i}\right) g\left(\nabla_{f_{i}} \xi, f_{j}\right)-g\left(\nabla_{f_{i}} \xi, f_{j}\right)^{2}
$$

combined with (5), (6) and (7) yields the following estimate for the scalar curvature of $H^{\prime}$.

(9)

$$
\begin{aligned}
\kappa_{H^{\prime}}= & \sum_{i, j=1}^{n-1} K_{H^{\prime}}\left(f_{i} \wedge f_{j}\right) \\
= & \sum_{i, j=1}^{n-p-1} K_{H^{\prime}}\left(f_{i} \wedge f_{j}\right)+2 \sum_{i, j=1}^{n-p-1} K_{H^{\prime}}\left(f_{i} \wedge f_{n-p}\right)+2 \sum_{i, j=1}^{n-p-1} \sum_{j=n-p+1}^{n-1}\left(f_{i} \wedge f_{j}\right) \\
= & (n-p-1)(n-p-2)\left[\frac{1}{\gamma^{2}\left(1+\gamma^{\prime 2}\right)}+O\left(\gamma^{-1}\right)\right] \\
& +2(n-p-1)\left[-\frac{\gamma^{\prime \prime}}{\gamma\left(1+\gamma^{\prime 2}\right)}+O\left(\gamma^{\prime \prime} \gamma\right)+O\left(\gamma^{-1}\right)\right]+O(1) \\
= & (n-p-1)(n-p-2) \frac{1}{\gamma^{2}\left(1+\gamma^{\prime 2}\right)} \\
& -2(n-p-1) \frac{\gamma^{\prime \prime}}{\gamma\left(1+\gamma^{\prime 2}\right)^{2}}+O\left(\gamma^{\prime \prime}\right)+O\left(\gamma^{-1}\right) .
\end{aligned}
$$

Now we can choose the curve $\gamma$ so that $\kappa_{H^{\prime}}$ is positive. After the initial bending, extend $\gamma$ as a straight line

$$
\gamma(t)=\gamma_{0}-\rho t, \quad t \leq t_{0} \leq r_{0}<\gamma_{0} / \rho
$$

as shown in Figure 4. 


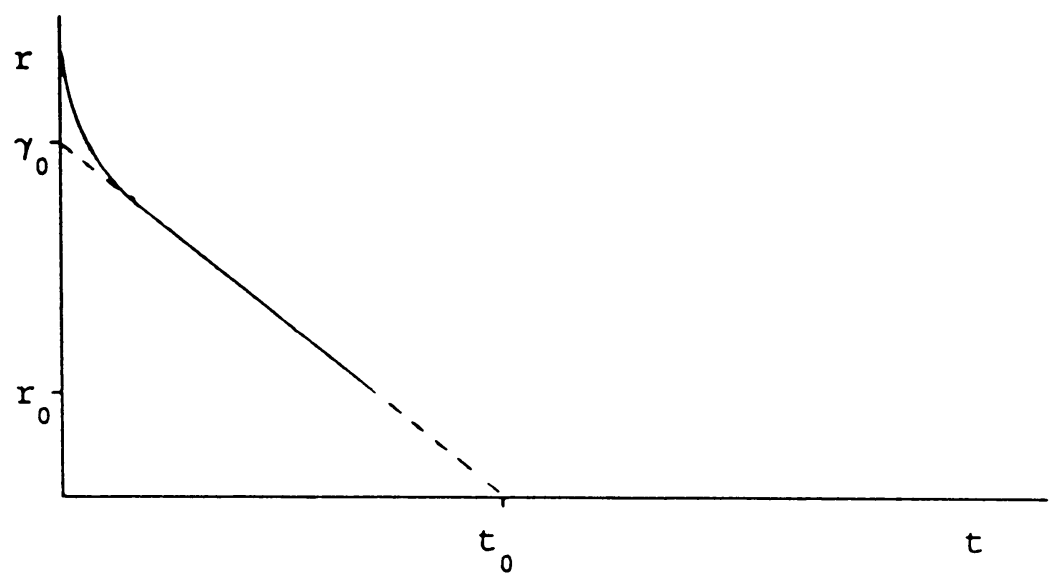

FIGURE 4

On the straight section

$$
\kappa_{H^{\prime}}=\frac{(n-p-1)(n-p-2)}{\left(\gamma_{0}-\rho t\right)^{2}\left(1+\rho^{2}\right)}+O\left[\frac{1}{\gamma_{0}-\rho t}\right],
$$

which can be made positive by choosing the parameter $\gamma_{0}$ small enough. Now bend $\gamma$ by choosing $\gamma^{\prime \prime}$ as in Figure 5 .

Note that $\gamma(t)>r_{0} / 2$ through this bending. From (9)

$$
\begin{aligned}
\kappa_{H^{\prime}} & =\frac{n-p-1}{\left(1+\gamma^{\prime 2}\right)}\left[\frac{n-p-2}{\gamma^{2}}-\frac{2 \gamma^{\prime \prime}}{\gamma\left(1+\gamma^{\prime 2}\right)}\right]+O\left(\gamma^{\prime \prime}\right)+O\left(\gamma^{-1}\right) \\
& \geq \frac{n-p-1}{\left(1+\gamma^{\prime 2}\right)}\left[\frac{n-p-2}{r_{0}^{2}}-\frac{2\left(\rho^{2} / 4 r_{0}\right)}{\left(r_{0} / 2\right)^{2}\left(1+(\rho-\rho / 8)^{2}\right)}\right]+O\left(\frac{1}{r_{0}}\right) \\
& =c \cdot \frac{1}{r_{0}^{2}}+O\left(\frac{1}{r_{0}}\right)
\end{aligned}
$$

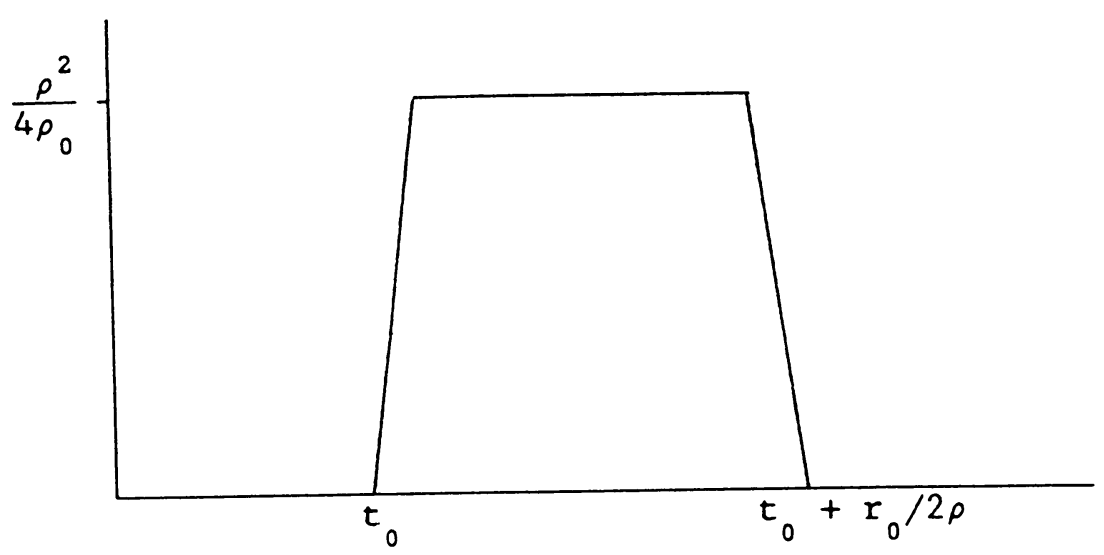

Figure 5 
where $c>0$ since $n-p-2 \geq 1$. So choosing the parameter $r_{0}$ sufficiently small will assure that $\kappa_{H^{\prime}}>0$ around this bend. At the end the slope of $\gamma$ has decreased by an amount

$$
\int_{t_{0}}^{t_{0}+r_{0} / 2 \rho} \gamma^{\prime \prime} \approx \frac{\rho}{8}
$$

so we can achieve $\gamma^{\prime}=0$ by repeating the above process 9 times.

This completes the demonstration that $U_{p-1}$ can be "pulled out" around a $p$-cell of $K$ while retaining positive scalar curvature on the boundary. Doing this for each $p$-cell constructs a regular neighborhood $U_{p}$ of the $p$-skeleton, as required.

2. Proof of Corollary 2. We construct $M$ as the boundary of a 2-complex in $\mathbf{R}^{5}$ follows. Let $K$ be a 2-complex with $\pi_{1}(K)=\pi$ and choose an embedding $\phi: K \rightarrow \mathbf{R}^{5}$. Let $U$ be a regular neighborhood of $\phi(k)$ and set $M=\partial U$. The long exact sequence for the pair $(U, M)$ shows that $\pi_{1}(M)=\pi$ and since the image $\phi(K) \subset \mathbf{R}^{5}$ is of codimension $\geq 3$ we know by Theorem 1 that $M$ has a metric of positive scalar curvature.

3. Proof of Theorem 3. Theorem 3 is proved as a corollary of Theorem 1 using the following construction. In the Riemannian product $M \times \mathbf{R}$ (standard metric on the $\mathbf{R}$-factor) consider the set $\tilde{K}=K \times[-1,1]$ made into a complex by letting the $k$-skeleton consist of the $k$-cells of $K \times\{-1\}$ and $K \times\{1\}$ (considered as copies of $K$ ) together with all cells of the form $\sigma \times[-1,1]$ where $\sigma$ ranges over all $(k-1)$-cells of $K, k=1, \ldots, n-q$.

The complex $\tilde{K}$ has codimension $q \geq 3$ in $M \times \mathbf{R}$ so by Theorem 1 it has a regular neighborhood $V$ with a metric of positive scalar curvature induced on $\partial V$. We can actually do a little better since the cell structure of $\tilde{K}$ and the method used to construct $V$ shows that we may actually choose $V$ so that $V \cap(-\delta, \delta)$ is a product $U \times(-\delta, \delta)$ for some $U \subset M$ and positive $\delta<1$. (See Figure 6 .) Now we simply note that $U$ is a regular neighborhood of $K$ diffeomorphic to $\partial V \cap M \times(0, \infty)$ and the metric on the latter has positive scalar curvature, product near the boundary.

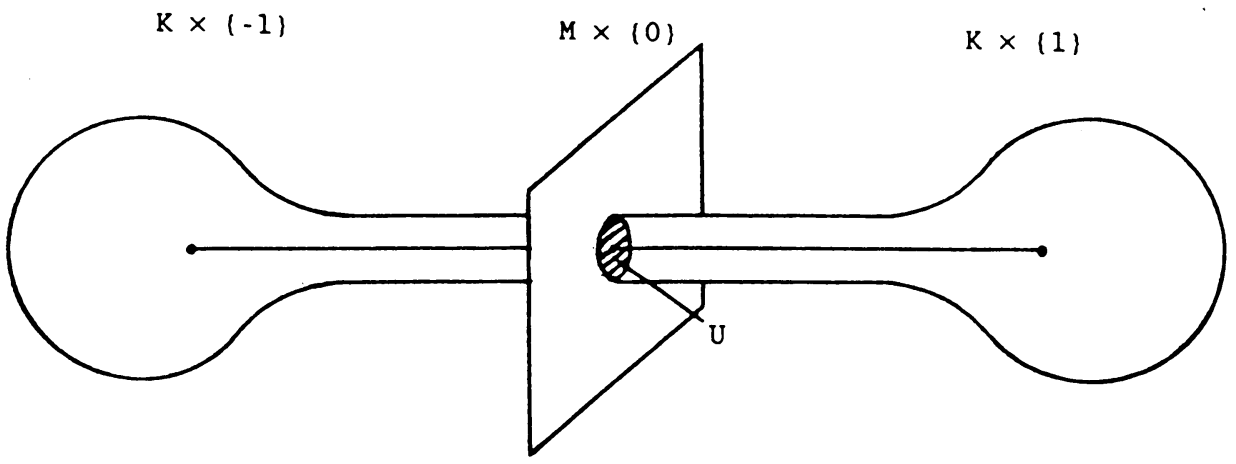

FigURE 6 
4. Proof of Theorem 4-The plumbing construction. Before outlining the plumbing construction we need the following lemmas.

LEMMA 10. Let $X$ be an $n$-dimensional manifold carrying a metric of positive scalar curvature, product near the boundary. For $n-r \geq 4$ let $H \cong D^{r} \times D^{n-r}$ be a solid $r$-handle attached to $X$ via a map $\phi: \partial D^{r} \times D^{n-r} \rightarrow \partial X$. Then $X \cup_{\phi} H$ also carries a metric of positive scalar curvature which is a product near the boundary.

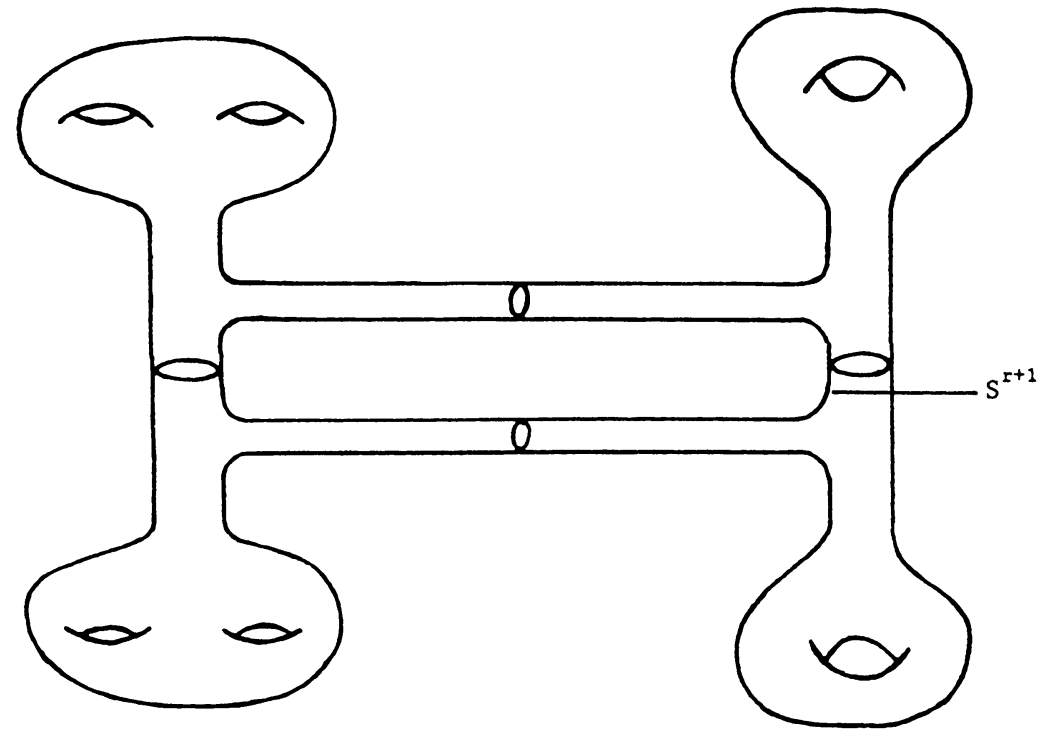

FIGURE 7

ProOF. Let $Y$ be the double of $X$ with $\sigma: Y \rightarrow Y$ the natural involution. There is a neighborhood of the join isometric to $\partial X \times(-\varepsilon, \varepsilon)$ with the product metric $d \sigma^{2}+d t^{2}$.

Fix an embedding $S^{r} \subset \partial X \times\{\varepsilon / 2\}$ and symmetrically "pull out" from small $\varepsilon / 4$-balls around it and its mirror image $\sigma\left(S^{r}\right) \subset \partial X \times\{-\varepsilon / 2\}$ while retaining positive scalar curvature. As in [GL] we can now construct $Y^{\prime}=Y$ with two $r$ dimensional surgeries equipped with a metric of positive scalar curvature. Also, by construction, the metric on $Y^{\prime}$ is still a product in a neighborhood of the join and $Y^{\prime}$ still admits an involution $\sigma^{\prime}: Y^{\prime} \rightarrow Y^{\prime}$ which is an isometry. See Figure 7. Now consider $S^{r+1} \subset Y^{\prime}$ formed $\sigma$-invariantly as

$$
S^{r+1}=S^{r} \times(-\varepsilon / 4, \varepsilon / 4) \cup D^{r+1} \cup D^{r+1}
$$

where $S^{r}$ is contained in the join and the $D^{r+1}$ 's are taken in the surgeries $D^{r+1} \times$ $S^{n-r+1}$ to be of the form $D^{r+1} \times$ \{point $\}$. Remove the $S^{r}$ and $\sigma$-invariantly deform the metric in an $\varepsilon / 8$-neighborhod being careful to keep the metric a product in an $\varepsilon / 8$-neighborhood of the join. We obtain $Y^{\prime \prime}=Y^{\prime}$ with an $(r+1)$-dimensional surgery which still carries positive scalar curvature and admits an involution $\sigma^{\prime \prime}$ which is an isometry. The metric is a product in an $\varepsilon / 8$-neighborhood of the join so the quotient $Y^{\prime \prime} / \sigma^{\prime \prime} \cong X \cup H$ has a metric of positive scalar curvature which is a product near the boundary. 
We only need the special case $r=0$ of Lemma 10. This allows us to form the connnected sum at the boundary of manifolds having metrics of positive scalar curvature which are products near the boundary while retaining such a metric on the result.

LEMMA 11. If metrics $g_{0}$ and $g_{1}$ on a compact manifold $M$ are in the same connected component of $R^{+}(M)$, that is, are homotopic through metrics of positive scalar curvature, then they are $H$-cobordant, that is, on the manifold $W=M \times[0,1]$ there is a metric $\bar{g}$ of positive scalar curvature, product near the boundary, with $\left.\bar{g}\right|_{M \times\{0\}}=g_{0}$ and $\left.\bar{g}\right|_{M \times\{1\}}=g_{1}$.

ProOF. Let $g:[0,1] \rightarrow R^{+}(M), g(0)=g_{0}, g(1)=g_{1}$ be a curve connecting $g_{0}$ and $g_{1}$ and pick a function $f_{R}:[0,1] \rightarrow \mathbf{R}^{+}$to have a graph as shown in Figure 8. Then the metric

$$
h=f_{R}(t) \cdot d t^{2}+g(t), \quad t \in[0,1]
$$

on $W$ will have positive scalar curvature for $R$ sufficiently large and by a slight deformation we may alter $h$ so that outside of $(\delta, 1-\delta) \times M$ it is a product.

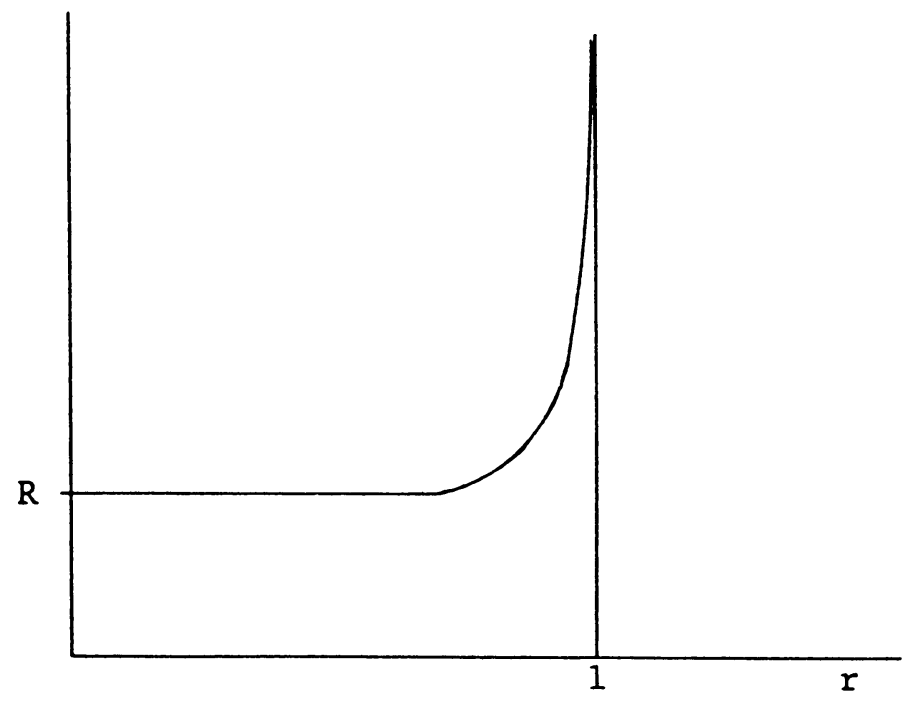

FIGURE 8

PLUMBing CONSTRUCTION. 1 . Let $G$ be a connected $\pi_{n-1}(S O(n))$-weighted graph with vertices $v_{i}, i=1, \ldots, L$, and corresponding weights $w_{i} \in \pi_{n-1}(S O(n))$.

(a) We form a connected $2 n$-dimensional manifold-with-boundary $X_{G}$ by plumbing the $D^{2 n}$-bundles over $S^{2 n}$ which correspond to the $w_{i}$. See [HNK] for details.

(b) The resulting manifold $X_{G}$ is diffeomorphic to a regular neighborhood of the skeleton formed by the base spheres $S_{i}$ of the plumbed bundles. By Theorem 3, for $n \geq 3, X_{G}$ admits a metric of positive scalar curvature which is a product near its boundary. 
(c) If $n$ is even, $n=2 m$, the intersection form of $X_{G}$ is

$$
\Phi_{G}=\left(\alpha_{i j}\right), \quad \alpha_{i j}= \begin{cases}e\left(w_{i}\right), & i=j, \\ 1, & i \neq j \text { and } \\ & v_{i} \text { connected to } v_{j} \text { by an edge of } G, \\ 0, & \text { otherwise. }\end{cases}
$$

Here $e\left(w_{i}\right)$ is the Euler number of the bundle $w_{i} \rightarrow S^{2 m}$.

(d) For $m \geq 2$ and $G$ a tree with $\Phi_{G}$ unimodular the resulting manifold $X_{G}$ has boundary $\partial X_{G}$ homeomorphic to a $(4 m-1)$-sphere. Let $\theta_{G}$ be the order of $\partial X_{G}$ in the group $\Theta_{4 m-1}$ of homeomorphic $(4 m-1)$-spheres.

2. Now suppose $G$ has connected components $G_{r}, r=1, \ldots, R$. As in 1 above:

(a) We can still form a connected manifold

$$
X_{G}=X_{G_{1}} \#_{\partial} \cdots \#_{\partial} X_{G_{R}}
$$

where here \# $\#_{\partial}$ means connected sum at the boundary.

(b) For $n \geq 3$ Lemma 11 and 1(b) above show that $X_{G}$ still carries a metric of positive scalar curvature which is a product near its boundary.

(c) For $n=2 m$ the intersection form $\Phi_{G}$ of $X_{G}$ has the form

$$
\Phi_{G}=\left(\begin{array}{lll}
\Phi_{G_{1}} & & \\
& \ddots & \\
& & \Phi_{G_{1}}
\end{array}\right) .
$$

Hence the signature of $X_{G}$ is

$$
\operatorname{sig}\left(X_{G}\right)=\sum_{r=1}^{n} \operatorname{sig}\left(X_{G_{r}}\right)
$$

and, in particular, if $R \cdot G$ denotes the graph consisting of $R$ disjoint copies of $G$ then

$$
\operatorname{sig}\left(X_{R \cdot G}\right)=R \cdot \operatorname{sig}\left(X_{G}\right) .
$$

(d) For $m \geq 2$ and each integer $q \geq 1$, if $G$ is a tree with $\Phi_{G}$ unimodular then the manifold $\partial X_{q \cdot\left(\theta_{G} \cdot G\right)}$ is diffeomorphic to the usual $(4 m-1)$-sphere.

3.

THEOREM 12. Fix $m \geq 2$. Let $G_{0}$ and $G_{1}$ be $\pi_{2 m-1}(S O(2 m))$-weighted graphs. Assume

(i) the intersection forms $\Phi_{G_{0}}$ and $\Phi_{G_{1}}$ are unimodular,

(ii) all weights $w$ have $p_{m / 2}(w)=0$, where $p_{m / 2}(w) \in H^{2 m}\left(S^{2 m}\right)$ is the $m / 2$ th Pontrjagin class of $w \rightarrow S^{2 m}$. Suppose $\partial X_{G_{0}}$ and $\partial X_{G_{1}}$ are diffeomorphic to some manifold $M$. Then the $\hat{A}$-genus of

$$
W \equiv X_{G_{0}} \cup(M \times[0,1]) \cup X_{G_{1}}
$$

is given by

$$
\hat{A}(W)=c\left(\operatorname{sig}\left(G_{0}\right)-\operatorname{sig}\left(G_{1}\right)\right)
$$

where $c$ is some nonzero constant. 
Proof. To calculate $\hat{A}(W)$ we use its expression in terms of the Pontrjagin classes $p_{k} \in H^{4 k}(W)$, that is,

$$
\hat{A}(W)=\hat{A}\left(p_{1}, \ldots, p_{m}\right)[W]
$$

where $\hat{A}\left(p_{1}, \ldots, p_{m}\right)$ is some universal homogeneous polynomial and $[W]$ is the fundamental class of $W$. Note that there is an explicit formula for the coefficients of $\hat{A}\left(p_{1}, \ldots, p_{m}\right)$ but we only need the fact that

$$
\hat{A}\left(p_{1}, \ldots, p_{m}\right)=c_{m} p_{m}+\text { terms involving only } p_{m-1}, \ldots, p_{1}
$$

where $c_{m}$ is some nonzero constant.

Compare this with the similar expression for the signature $\operatorname{sig}(W)$ of $W$

$$
\operatorname{sig}(W)=L\left(p_{1}, \ldots, p_{m}\right)[W]
$$

where $L\left(p_{1}, \ldots, p_{m}\right)$ is the Hirzebruch $L$-genus. Again all we need to know concerning the exact formula for $L$ is that

$$
L\left(p_{1}, \ldots, p_{m}\right)=d_{m} p_{m}+\text { terms involving only } p_{m-1}, \ldots, p_{1}
$$

where $d_{m}$ is some nonzero constant. We also have an expression for $\operatorname{sig}(W)$ directly in terms of the signatures $\operatorname{sig}\left(G_{0}\right)$ and $\operatorname{sig}\left(G_{1}\right)$ of $X_{G_{0}}$ and $X_{G_{1}}$ :

$$
\operatorname{sig}(W)=\operatorname{sig}\left(G_{1}\right)-\operatorname{sig}\left(G_{0}\right) .
$$

We now show that in our case all the lower Pontrjagin classes $p_{k}, k=1, \ldots, m-1$, of $W$ are zero. Using the Mayer-Vietoris sequence with $X_{G_{0}} \cup X_{G_{1}} \underset{\text { h.e. }}{\sim} W$ and $X_{G_{0}} \cap X_{G_{1}} \underset{\text { h.e. }}{\sim} M$ we obtain $H^{4 k}(W)=0$ except possibly in the case that $m$ is even, $m=2 l$, and $k=1$. For the exceptional case it suffices to evaluate $p_{1}\left[S_{i}\right]$ where the $S_{i}$ are the base spheres of the plumbed bundles which together form a basis for $H_{4 l}(W)$. The integer $p_{1}\left[S_{i}\right]$ just depends on the bundle $\left.T W\right|_{S_{i}} \rightarrow S_{i}$ which splits as

$$
\left.T W\right|_{S_{i}}=T S_{i} \oplus N S_{i} .
$$

Here $N S_{i} \rightarrow S_{i}$ is the normal bundle of $S_{i}$ in $W$ which, by construction, is just $w_{i} \rightarrow S_{i}$, where $w_{i}$ is the weight assigned to the vertex corresponding to $S_{i}$. By (i) $p_{1}\left(w_{i}\right)=0$. Hence

$$
\begin{aligned}
p_{1}\left[S_{i}\right] & =p_{1}\left(T W \mid S_{i}\right)\left[S_{i}\right]=p_{1}\left(T S_{i} \oplus N S_{i}\right)\left[S_{i}\right] \\
& =p_{1}\left(T S_{i} \oplus w_{i}\right)\left[S_{i}\right]=p_{1}\left(T S_{i}\right)\left[S_{i}\right]+p_{1}\left(w_{i}\right)\left[S_{i}\right]=0 .
\end{aligned}
$$

The theorem now follows:

$$
\begin{aligned}
\hat{A}(W) & =\left(c_{m} p_{m}\right)[W], \quad c_{m} \neq 0, \\
& =\left(c_{m} / d_{m}\right) \operatorname{sig}(W), \quad d_{m} \neq 0, \\
& =c\left(\operatorname{sig}\left(G_{0}\right)-\operatorname{sig}\left(G_{1}\right)\right), \quad c \neq 0 .
\end{aligned}
$$

4. Let $E_{8}$ be the tree

$$
\begin{array}{lllllll}
v_{1} & v_{2} & v_{3} & v_{4} & v_{5} & v_{6} & v_{7} \\
\hline & l_{v_{8}}
\end{array}
$$


with each vertex weighted by $w_{T S}$, the classifying element of the tangent bundle $T S \rightarrow S^{2 m}$. From 1(c) we find that the associated intersection form is the wellknown signature 8 unimodular form

$$
E_{8}=\left(\begin{array}{llllllll}
2 & 1 & 0 & 0 & 0 & 0 & 0 & 0 \\
1 & 2 & 1 & 0 & 0 & 0 & 0 & 0 \\
0 & 1 & 2 & 1 & 0 & 0 & 0 & 0 \\
0 & 0 & 1 & 2 & 1 & 0 & 0 & 0 \\
0 & 0 & 0 & 1 & 2 & 1 & 0 & 1 \\
0 & 0 & 0 & 0 & 1 & 2 & 1 & 0 \\
0 & 0 & 0 & 0 & 0 & 1 & 2 & 0 \\
0 & 0 & 0 & 0 & 1 & 0 & 0 & 2
\end{array}\right) .
$$

Hence, from 2(b), (c), and (d), for $m \geq 2$ and each $q \geq 1$

(i) the corresponding manifolds $X_{q \cdot\left(\theta_{E_{8}} \cdot E_{8}\right)}$ have metrics of positive scalar curvature which are products near their boundaries.

(ii) $\operatorname{sig}\left(X_{q \cdot\left(\theta_{E_{8}} \cdot E_{8}\right)}\right)=q\left(\theta_{E_{8}} \cdot 8\right)$,

(iii) the boundaries $\partial X_{q \cdot\left(\theta_{E_{8}} \cdot E_{8}\right)}$ are diffeomorphic to the usual $(4 m-1)$-sphere. So for $m \geq 2$ and $q_{0} \neq q_{1}$ we have from Lemma 10 that if the metrics on $\partial X_{q_{0} \cdot\left(\theta_{E_{8}} \cdot E_{8}\right)}$ and $\partial X_{q_{1} \cdot\left(\theta_{E_{8}} \cdot E_{8}\right)}$ are in the same connected component of $R^{+}\left(S^{4 m-1}\right)$ then

$$
W \equiv X_{q_{0} \cdot\left(\theta_{E_{8}} \cdot E_{8}\right)} \cup S^{4 m-1} \cup X_{q_{1} \cdot\left(\theta_{E_{8}} \cdot E_{8}\right)}
$$

admits a metric of positive scalar curvature. However, from Theorem 12,

$$
\begin{aligned}
\hat{A}(W) & =c\left(q_{0}\left(\theta_{E_{8}} \cdot 8\right)-q_{1}\left(\theta_{E_{8}} \cdot 8\right)\right) \\
& =\left(q_{0}-q_{1}\right) c^{\prime}, \quad c^{\prime} \neq 0, \\
& \neq 0
\end{aligned}
$$

and thus $W$ cannot carry positive scalar curvature. This contradiction shows that the metrics fall into different connected components of $R^{+}\left(S^{4 m-1}\right)$, which completes the theorem.

\section{REFERENCES}

[GL] M. Gromov and H. B. Lawson, The classification of simply connected manifolds of positive scalar curvature, Ann. of Math. 111 (1980), 423-434.

[HNK] F. Hirzebruch, W. D. Neumann and S. S. Koh, Differentiable manifolds and quadratic forms, Lecture Notes in Pure and Appl. Math., Vol. 4, Deker, 1971.

[SY] R. Schoen and S. T. Yau, On the structure of manifolds with positive scalar curvature, Manuscripta Math. 28 (1979), 159-183. 48109

Department of Mathematics, University of Michigan, AnN ARbor, Michigan

('urrent address: School of Mathematics and Computer Studies, SAIT, Levels Campus, The Levels, SA 5095, Australia 